\begin{tabular}{|c|c|c|c|c|c|c|c|c|}
\hline \multirow[t]{2}{*}{ Study ID } & \multirow{2}{*}{$\begin{array}{l}\text { FEV1 } \\
\text { (\% predicted) }\end{array}$} & \multirow[t]{2}{*}{ On PPI } & \multirow{2}{*}{$\begin{array}{l}\text { RESQ-7 } \\
\text { Heartburn } \\
\text { Score }^{1} \\
(0-5)\end{array}$} & \multicolumn{3}{|l|}{ Impedance results } & \multicolumn{2}{|l|}{$\mathrm{pH}$ results } \\
\hline & & & & $\begin{array}{l}\text { Number of episodes } \\
\text { (impedance) }\end{array}$ & Proximal & Supine & Acid exposure & Longest reflux episode \\
\hline Normal values & & & & $<75$ & $<30$ & $<14$ & $<4.2 \%$ & $<9.2 \mathrm{mins}$ \\
\hline 001 & 60 & No & 2.2 & $\underline{323.1}$ & $\underline{96.8}$ & $\underline{56.6}$ & $\underline{21}$ & $\underline{77.9}$ \\
\hline 003 & 50 & Yes & 1.2 & $\underline{118.8}$ & 22.1 & $\underline{27.7}$ & 3.6 & 7.4 \\
\hline 005 & 63 & Yes & 0.8 & 51 & 19.7 & 4.6 & 10.7 & $\underline{16.8}$ \\
\hline 006 & 43 & Yes & 0.2 & 67.6 & 11.3 & 3.1 & 1.2 & 7.4 \\
\hline 007 & 53 & Yes & 3.2 & 153.9 & $\underline{66.3}$ & $\underline{15}$ & 14.2 & $\underline{66.5}$ \\
\hline 009 & 26 & Yes & 4 & 154.9 & $\underline{50.5}$ & $\underline{25.8}$ & $\underline{7}$ & $\underline{45.8}$ \\
\hline 010 & 26 & No & 0 & $\underline{89.3}$ & 16.3 & $\underline{15.3}$ & $\underline{4.5}$ & 16.8 \\
\hline 011 & 72 & Yes & 0.96 & $\underline{83.6}$ & 15.9 & 6.4 & 10.7 & $\underline{34.5}$ \\
\hline 012 & 39 & Yes & 0 & 192.7 & $\underline{71.5}$ & NA & NA & NA \\
\hline 014 & 82 & Yes & 0 & $\underline{82.3}$ & 3.3 & 9.9 & 2.9 & $\underline{16.9}$ \\
\hline
\end{tabular}

1 - This is the score for severity of heartburn, which is a single domain of the RESQ-7.

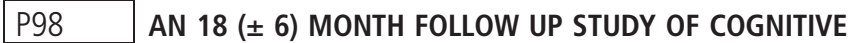 FUNCTION IN ADULTS WITH CYSTIC FIBROSIS RELATED DIABETES (CFRD)}

${ }^{1}$ HK Chadwick, ${ }^{2} \mathrm{~A}$ Morton, ${ }^{1} \mathrm{~L}$ Dye, ${ }^{1} \mathrm{CL}$ Lawton, ${ }^{2} \mathrm{MW}$ Mansfield, ${ }^{2} \mathrm{D}$ Peckham. ${ }^{1}$ School of Psychology, University of Leeds, Leeds, UK; ${ }^{2}$ Regional Adult Cystic Fibrosis Unit, St James' Hospital, Leeds, UK

\subsection{6/thoraxjnl-2016-209333.241}

Introduction and objectives Cognitive impairments have been observed in people with type 1 and 2 diabetes. People with cystic fibrosis (CF) who have developed CF related diabetes (CFRD) also show some degree of impairment relative to healthy controls. The aim of this study was to examine cognitive function in people with CFRD after an $18( \pm 6)$ month period to assess any change in performance.

Method Adult ( $>16$ years old), pancreatic insufficient patients with insulin treated CFRD registered to a large UK CF unit who had adequate verbal and written English were eligible. Cognitive performance was assessed using parallel versions of tests from the Cambridge Neuropsychological Test Automated Battery (CANTAB). Subjective ratings of sleep, stress, mood, cognitive performance and minor daily cognitive errors were also collected. At baseline, 67 people were tested; 49 non transplanted and 18 post transplantation patients. Twelve patients were lost to follow-up. To date, 43 people have been retested after an $18( \pm 6)$ month period; 35 non transplanted and 8 post transplantation patients.

Results At follow up, blood glucose levels were significantly lower during testing (reflecting better glycaemic control in some patients) and people reported significantly fewer occurrences of minor daily cognitive errors within the past 6 months. There was no change in performance between baseline and follow up on tests of motor function, visual memory and new learning, immediate pattern recognition, working memory and mental flexibility. On tests of immediate and delayed verbal memory, delayed pattern recognition and processing speed, performance had significantly improved at follow up.

Conclusion Cognitive function is impaired in people with CFRD but remains stable over an $18( \pm 6)$ month period. Deficits in cognitive performance may impact upon quality of life and ability to adhere to treatment.

\section{P99 CYSTIC FIBROSIS MEDICATIONS AT TRANSFER FROM PAEDIATRIC TO ADULT CARE - WHAT ARE PATIENTS ACTUALLY TAKING?}

1J Strange, ${ }^{2} \mathrm{~K}$ Cox, ${ }^{2} \mathrm{~A}$ Jones. ${ }^{1}$ University of Manchester, Manchester, UK; ${ }^{2}$ Manchester Adult CF Centre, Manchester, UK

\subsection{6/thoraxjnl-2016-209333.242}

Introduction and objectives Cystic fibrosis (CF) is a multi-system disease requiring complex, high-burden treatment regimens. Discrepancies can arise between hospital and GP drug lists, in turn impacting patient's access to required long-term medications. One large adult CF centre has been conducting medicines reconciliation at the point of transition from paediatric care, with the goal of identifying and eliminating such discrepancies.

The objective of this study was to evaluate the accuracy of hospital medication lists versus the GP repeat prescription lists, at the point of transition from paediatric to adult care.

Methods Drug lists were assessed for discrepancies, in drug or dose, as well as non-collection of items. These issues were subgrouped to evaluate whether frequency varied by drug class.

Results Drug list from 99 patients from a 4.5-year period (20112016) were included, featuring a total of 1201 items, with a mean of 12 , range $1-22$, items per patient. There was a drug discrepancy in $11.7 \%$ of total items, with greatest frequency occurring in inhaled and nebulised antibiotics (20.0\%), as seen in Figure 1. There was a dose discrepancy in $3.9 \%$ of total items, with greatest frequency occurring in oral antibiotics $(8.7 \%)$. It was also found that $9.2 \%$ of items had not been collected from GP for $\geq 6$ months, with greatest frequency occurring in nebulised medicines $13.8 \%$.

Conclusions Discrepancies are common across CF medication lists. These may have a detrimental impact on clinical care, as patients are unable to access the required medicines from their GP, and hospitals may prematurely escalate care. Furthermore, a number of items prescribed by GPs are not regularly collected, indicating poor adherence. Medicines reconciliation at transition of care has a significant impact in identifying these issues, and CF centres should consider whether to also routinely include this process during CF annual reviews. 